\title{
Genetic Counseling and Testing for Colorectal Cancer in Young Adults: Mini-Review
}

\author{
Ethem Unal*, Sema Yuksekdag, Abdullah Yildiz, Ozgul Duzgun, Ahmet Topcu, M Taha Demirpolat, Omer Faruk \\ Ozkan
}

Department of General Surgery, Umraniye Training and Research Hospital, Health Sciences University, Istanbul, Turkey

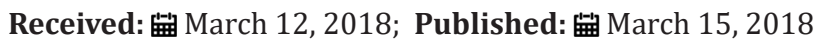

*Corresponding author: Ethem Unal, MD, Associate Professor of Surgery, Tavukcuyolu Caddesi, Umraniye, Istanbul, Turkey

\begin{abstract}
Colorectal cancer (CRC) has one of the largest proportions of familial cases. Two to $5 \%$ of all colon cancers arise in the setting of inherited syndromes, including Lynch syndrome (hereditary nonpolyposis colorectal cancer [HNPCC]), familial adenomatous polyposis (FAP), attenuated FAP, MUTYH-associated polyposis (MAP), and certain hamartomatous polyposis conditions like Peutz-Jeghers syndrome (PJS) and juvenile polyposis syndrome (JPS). All of these conditions are inherited, autosomal dominant disorders, except MAP, which is autosomal recessive [1]. Although clinical similarities do exist, each has different cancer risks, characteristic clinical features, and separate genetical etiologies. In addition to these syndromes, up to $30 \%$ of colon cancers exhibit increased familial risk, likely related to inheritance. A number of less penetrant, but possibly more frequent susceptibility genes have been identified for this level of inheritance. Determination of predisposing genes allows for accurate risk assessment and more precise screening approaches. Examples include common polymorphisms in genes that regulate metabolism or genes that are regulated by environmental or other genetic factors.
\end{abstract}

Abbreviations: CRC: Colorectal cancer; HNPCC: Nonpolyposis Colorectal Cancer; FAP: Familial Adenomatous Polyposis; MAP: MUTYHAssociated Polyposis; JPS: Juvenile Polyposis Syndrome.

\section{Introduction}

Lynch syndrome is the result of a germline mutation in a class of hMSH2, hMLH1, hMSH6, and hPMS2. The MMR system is and insertion-deletion loops that form during DNA replication. Germline mutations in genes involved in DNA MMR (necessary for maintaining genomic stability by correcting single-base mismatches), including hMSH2 and hMLH1 account for the up to $90 \%$ of Lynch syndrome cases; mutations in hMSH6 account for approximately 10\% and mutations in hPMS2 are detected on rare occasions [2]. FAP (development of hundreds to thousands of colonic adenomas, beginning in early adolescence, and inevitable CRC in untreated individuals by 39 years), attenuated FAP (70\% lifetime risk of CRC, an average of approximately 30 colonic adenomatous polyps), and Gardner syndrome (FAP with osteomas, epidermoid cysts, dental disorders, and/or desmoid tumors) all result from mutations in gene APC, which encodes a tumor suppressor that is part of the WNT signaling pathway.MAP is caused by biallelic mutations in MUTYH (MYH;part of the base-excision repair pathway, which is involved in defending against oxidative DNA damage) and is characterized by the presence of adenomatous polyposis of colo-rectum, and an increased risk of especially proximal colonic neoplasms, like attenuated FAP [3]. PJS and JPS are hamartomatous polyposis diseases hat are both associated with an increased risk for CRC and other malignancies. Gastrointestinal symptoms first occur in the early teenage years and include small bowel obstruction and intussusception. Mutations in STK11 (LKB1) are the only known cause of PJS, whereas JPS is caused by mutations in either SMAD4 or BMPR1A [4]. Another known very rare hamartomatous condition, Cowden syndrome, arises from mutations of PTEN [5].

Identification of the genes that cause these colon cancer syndromes has led to the development of specific management guidelinesand genetic teststhat can diagnose these familial disorders. These guidelines are important, not only for the affected patient, but also for their family members. If a patient has FAP, close family members should be tested early on, usually as teenagers. This is because CRC in patients with FAP tends to arise at a very young age. If a patient has Lynch syndrome, family members can wait until 
they are about 20 to 25 years old to undergo genetic testing. At-risk patients can be offered genetic counseling and testing to determine whether they carry a detectable mutation for such a syndrome. If so, this information provides the clinician with valuable data about the patient's risk for other cancers, and alerts surgeon about the correct time for surgery.

\section{Conclusion}

In conclusion, genetic testing is available for most hereditary CRC syndromes and can be used to confirm suspected diagnosis, to clarify risks of extra-colonic cancers in affected individuals, and to identify relatives who are also at risk. Therefore, genetics analyses of $\mathrm{CRC}$ risk need to be included in mainstream clinical practice.

\section{References}

1. Jasperson KW, Tuohy TM, Neklason DW, Burt RW (2010) Hereditary and familial colon cancer. Gastroenterology 138(6): 2044-2058.

2. Peltomaki P, Vasen H (2004) Mutations associated with HNPCC predisposition-Update of ICG-HNPCC/INSiGHT mutation database. Dis Markers 20(4): 269-276.

3. Lubbe SJ, Di Bernardo MC, Chandler IP, Houlston RS (2009) Clinical implications of the colorectal cancer risk associated with MUTYH mutation. J Clin Oncol 27(24): 3975-3980.

4. Schreibman IR, Baker M, Amos C, McGarrity TJ (2005) The hamartomatous polyposis syndromes: a clinical and molecular review. Am J Gastroenterol 100(2): 476-490.

5. Gammon A, Jasperson K, Kohlmann W, Burt RW (2009) Hamartomatous polyposis syndromes. Best Pract Res Clin Gastroenterol 23(2): 219-231.

\section{(C) This work is licensed under Creative}

To Submit Your Article Click Here:

Submit Article

DOI: $10.32474 /$ PRJFGS.2018.01.000109

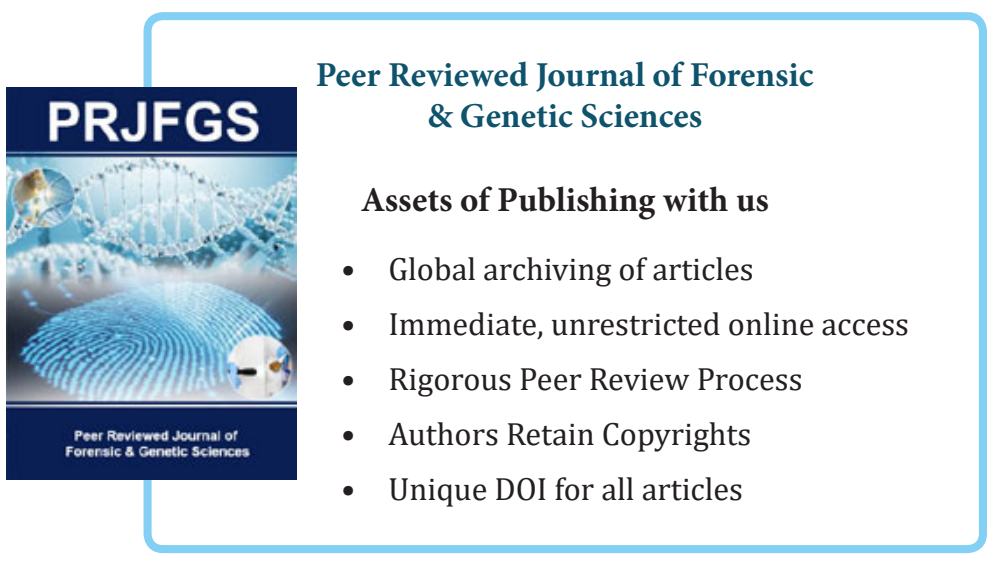

